\title{
CENTRIFUGAL AND CENTRIPETAL THINKING ABOUT THE BIOPSYCHOSOCIAL MODEL IN PSYCHIATRY
}

\author{
Kathryn Tabb ${ }^{1}$ \\ ${ }^{1}$ Philosophy Program, Bard College
}

Original scientific article—Received: 25/07/2021 Accepted: 21/09/2021

\begin{abstract}
The biopsychosocial model, which was deeply influential on psychiatry following its introduction by George L. Engel in 1977, has recently made a comeback. Derek Bolton and Grant Gillett have argued that Engel's original formulation offered a promising general framework for thinking about health and disease, but that this promise requires new empirical and philosophical tools in order to be realized. In particular, Bolton and Gillett offer an original analysis of the ontological relations between Engel's biological, social, and psychological levels of analysis. I argue that Bolton and Gillett's updated model, while providing an intriguing new metaphysical framework for medicine, cannot resolve some of the most vexing problems facing psychiatry, which have to do with how to prioritize different sorts of research. These problems are fundamentally ethical, rather than ontological. Without the right prudential motivation, in other words, the unification of psychiatry under a single conceptual framework seems doubtful, no matter how compelling the model. An updated biopsychosocial model should include explicit normative commitments about the aims of medicine that can give guidance about the sorts of causal connections to be prioritized as research and clinical targets.
\end{abstract}

Keywords: Biopsychosocial model; precision medicine, medical ethics; philosophy of psychiatry 


\section{Introduction}

Writing on the tortured status of psychiatric classification, Scott Lilienfeld (2014) characterized the Diagnostic and Statistical Manual of Mental Disorders (DSM) as buffeted about by conflicting centrifugal and centripetal forces. Often psychiatric nosology is envisioned in awkward suspension between the twin stars of Snow's two cultures. Lilienfeld's metaphor has it instead shifting unstably amidst the ongoing negotiations of a range of subtler powers. For my purposes I will borrow the metaphor not - or not just - in order to reflect on the shaky orbit of the DSM around the nebula of scientific validity, but rather in order to say something about the shifting conceptual structure of the discipline of psychiatry as a whole. The centripetal forces I am interested in are those compressing the field of psychiatry into some sort of conceptual unity. The centrifugal ones are those pulling it apart, as some bits spin off into the basic and applied sciences, and others move farther into humanistic spaces like psychotherapeutics, recovery movements, and social welfare projects. Going back to Jaspers, a worry that psychiatry has two distinct projects that are increasingly uneasy together-one that values explanation, and one that values understanding - has driven scholars and clinicians to offer up various pleas for centripetalism, the calling back to order of an undisciplined discipline. I am thinking of titles like David Brendel's Healing Psychiatry, or Tanya Luhrmann's Of Two Minds. Many of these centripetal pleas attribute this historic split to the broader Cartesian severing of the ontological into the physical and the mental, which, they claim, has destabilized psychiatry, balanced as it is on the point where the two meet.

Perhaps most notable among such attempts has been the biopsychosocial model, introduced by George L. Engel in 1977. If it still functions as a model for psychiatry - rather than as something more like a zeitgeist-it does so in an optative mood; not so much supplying a rigorous descriptive or prescriptive representation of contemporary medicine as offering a cultivated and relatively benign rebuke to the way things are. In their monograph The Biopsychosocial Model of Health and Disease: New Philosophical and Scientific Developments, Derek Bolton and Grant Gillett aim to realize some of the model's original transformative potential, not only for psychiatry but for medicine writ large. Through integrating not only our best contemporary theories of each level of analysis - the biological, the psychological, and the social — but also our best theories of their concomitance, the authors aim to save the model from the aggregated charges of imprecision, disappointing scientific validity, and philosophical incoherence that have built up over decades (Bolton and Gillett 2019, v). 
I am sympathetic to the anxieties about centrifugalism that have increasingly animated philosophers of psychiatry. I am also galvanized by Bolton and Gillett's case for drawing our attention back to the biopsychosocial model's original promise, on the grounds that we now have the scientific and philosophical tools to make it work better. In response, I want to offer some reasons for thinking that the centripetal force that Bolton and Gillett posit - a fundamentally metaphysical force-may not sufficiently address some of prevalent worries about psychiatry's current predicaments (I think it is the case that these worries are also applicable to much of contemporary medicine, such that the shortcomings I see in their model would apply in other contexts as well, but here I limit my discussion to psychiatry). In particular, I will argue that ethical arguments for centripetalism are necessary alongside metaphysical ones, and that therefore, if the biopsychosocial model is to be resuscitated, it should be resuscitated in a manner that gives ethical forces primacy. I will not, for the most part, engage with the details of Bolton and Gillett's argument, which I think are rich and exciting, and which I expect will prompt a great deal of interest from philosophers working at the interstices of explanation, causation, and philosophy of mind. Little I say here conflicts with the nuts and bolts of their new model, but I do want to shift the center of gravity a bit.

In the following section I give a brief synopsis of Bolton and Gillett's project, a true challenge given the density and richness of their slim book. In Section 3 I will review what I see as the main forces working against conceptual unity in psychiatry, and review the strongest grounds, as I understand them, for worries that the discipline increasingly lacks a clearly delineated conceptual core. I will argue that this is less about dualismindeed, less about philosophy! - than about historic, economic, and sociocultural factors which have motivated different practitioners to adopt different competing conceptual schemata. In particular I will highlight the dramatic rise of professional specialization within the field of psychiatry during the twentieth century, and the related dominance of translational science over clinical science within psychiatric biomedicine. In Section 4 I will discuss how a focus on bioethics could complement the new biopsychosocial model by guiding choices about which causal relationships should be prioritized as research targets in psychiatry. Finally, I will conclude with some reflections on what it might look like to integrate ethical principles into the new biopsychosocial model such that they, too, would act as a centripetal force. 


\section{Ontological Centrifugalism, Ontological Centripetalism}

Bolton and Gillett's case must start with persuasive evidence that the biopsychosocial model is worth restoring. Their project responds to critics like Nassir Ghaemi, who frames his Rise and Fall of the Biopsychosocial Model around the arresting claim that the model in its original psychiatric context "rose from the ashes of psychoanalysis and is dying on the shoals of neurobiology" (2010, ix). Engel's intended intervention indeed arose from the contingencies of its historical moment - by the nineteen seventies the conflagration, or sea change, from the old psychoanalytic paradigm that had shaped the first edition of the DSM in 1952 to the operationalism that guiding the production of the DSM-III (1980) was well underway. The optimism over psychiatry's status as a science, which led to the emphasis on objective observation in the manual's third edition, was due in part to recent discoveries of powerful new psychotropic drugs. While these advances were not, actually, born of new insights into the causal mechanisms underlying mental illness, they gave reason to hope that scientific breakthroughs would be forthcoming. Engel's biopsychosocial model was intended to counter the rising enthusiasm for defining disease exclusively in terms of "somatic parameters", not only in psychiatry but in medicine as a whole (Engel 1992, 317). At a time when many psychiatrists were desperate to justify psychiatry as a legitimate medical science even as the care of the mentally ill was increasingly handled by practitioners without MD's, Engel's intervention had a ready-made constituency in those for whom the radicalism of the antipsychiatrists and the absolutism of the biomedicalists were both unpalatable. Instead of seeking to force psychiatry into the existing medical paradigm, Engel $(1992,320)$ aimed to use psychiatry's incoherence as a wedge to transform medicine as a whole, by showing that its central commitment to the biomedical model was no more than dogma.

Engel attributed the ideological nature of biomedicine, which he characterized as an allegiance to a reductionist, physicalist treatment of disease states as biological dysfunctions, to broad trends in intellectual history. "With mind-body dualism firmly established under the imprimatur of the Church," he wrote,

classical science readily fostered the notion of the body as a machine, of disease as the consequence of breakdown of the machine, and of the doctor's task as repair of the machine. Thus, the scientific approach to disease began by focusing in a fractional-analytic way on biological (somatic) processes and ignoring the behavioral and psychosocial. (Engel 1992, 321) 
The biopsychosocial model aims to counter this influential philosophical dogma by integrating an understanding of the patient's psychosocial context, including their broader healthcare context. For Engel, this approach was a crucial corrective not just for psychiatry but for medicine as a whole. The exclusion of "mental substance" (or its modern analogs) caused, in his view, a general crisis for not only clinical but also for scientific understanding (Engel 1980, 103). Engel's professional passions, over the course of his career, came to focus on the integration of personlevel explanations into our understanding of such quintessentially somatic conditions as heart disease (Ghaemi 2010, 44). As such, his presentation of the biopsychosocial model is primarily addressed to the general physician, and makes the case for treating social factors as relevant to every case of medical decision-making.

Bolton and Gillett agree with Engel's emphasis on the distorting influence of Cartesian dualism, but also agree with critics like Ghaemi who think that his proposed solution - of a general biopsychosocial model-is too vague and unsatisfactory with respect to the scientific details and the philosophical framework (indeed, Ghaemi has argued that the model ultimately has centrifugal, rather than centripetal, effects because of its milquetoast metaphysics, which he believes amounts only to a vapid sort of pluralism). Bolton and Gillett believe, however, that critics err in looking to the model itself to fill in the specifics, which should instead be gathered empirically for each specific stage of each specific health condition. "In this sense", the authors write, "there are multiple specific biopsychosocial models" (Bolton and Gillett 2019, 15); one might think they are too modest here, insofar as their account actually gives rise to countless new models! They are quick to correct the idea, however, that they are therefore pushing for (in the language of this paper) centrifugalism, emphasizing that a general model is still needed. Only a unifying framework can provide the "foundational theoretical constructs" that medicine needs. These theoretical constructs, in Bolton and Gillett's view, are "the ontology of the biological, the psychological, and the social-and especially the causal relations within and between these domains" $(2019,19)$. In other words, they are replacing the "massive historical baggage, carried in the long history of physicalism, dualism and reductionism" with a modern metaphysics that can ground the collected scientific findings of biomedical research. For medical findings, the authors argue, simply are biopsychosocial. What will unify medicine, countering the outward push of the vestiges of dualism, is a new theoretical framework recognizing these more inclusive ontological facts, and providing theoretical tools, like a new theory of causation that allows for not only bottom-up but also top-down causation. 
Engel was also interested in the role of medical ontology in grounding the biopsychosocial model, and drew on the systems theory in vogue at the time he was writing. For Bolton and Gillett, the new tenets of biopsychosocial causation are to be grounded in modern theories of information-based regulatory control-here they go back to the work of Schrödinger to ground their account of biological systems via an antireductionist biophysics. They also broaden their exploration of topdown causation to include personal agency as a core function of psychology that in turn impacts the biological. The body, therefore, can be "characterised not in mechanical terms, but in terms of functional processes involving information control" (Bolton and Gillett 2019, 79); in the production and management of information, the mental and the physical are "entangled".

I want here to emphasize the close connection in Bolton and Gillett's project between the causes of centrifugalism they attribute to medical theory-physicalism, dualism, and reductionism-and their favored metaphysical counterforce. Like other critics of the biopsychosocial model, the authors emphasize the powerlessness of the model if its content is allowed to be shaped by the weight of a problematic philosophical tradition. When emphasizing that the task of their new model is "defining biopsychosocial ontology and causation," they note

the special need for this because [of] the deeply entrenched assumptions of physicalism, dualism and reductionism that have been so influential in the development of the life and human sciences. (Bolton and Gillett 2019, 138)

They believe that that tradition has caused medical researchers to neglect the pursuit of certain scientific facts, namely those that require a nondualistic, non-physicalist, or non-reductive ontology: "With these assumptions, only physical properties and causation appear real, while the mind is a non-causal epiphenomena [sic], and social organization and processes can hardly be comprehended at all" $(2019,138)$. Accordingly, their project aims to not only provide the missing ontology, but to argue that the biopsychosocial model must contain such an undergirding conceptual structure if medical facts are to be legible to scientists.

In the following section I will argue that post-Cartesian philosophy, while a distal cause, is not the most immediate centrifugal pressure on at least one branch of medicine where it is often cited: psychiatry. Engel himself acknowledged the general point, writing, "The power of vested interests, social, political, and economic, are formidable deterrents to any effective assault on biomedical dogmatism" $(1992,328)$. Bolton and Gillett pay 
nuanced and generative attention to the role of autonomy and recognition in the individual's encounter with their social worlds, but they group such factors under the social arm of their unified model, and therefore approach them from a metaphysical perspective. I agree with the authors that the social, political, and economic forces driving biomedicalism are powerful, but I argue in the following section that there is little reason to think they will be attenuated by the introduction of the "right" metaphysics. This is not because most advocates of biomedicine are committed to the view that the mind or social organization and its processes are insubstantial, epiphenomenal, or incomprehensible; it is that they do not find these levels of explanation relevant to medicine's most rewarding projects. After explaining how these non-philosophical forces operate in psychiatry in the following section, I show that while Bolton and Gillett's model can offer a valuable corrective to them, it is ethical counterforces that are more likely to take hold.

\section{Centrifugalism in Psychiatry: Other Sources}

I have no doubt that philosophical concepts have been crucial to psychiatry's evolving self-image. Alongside the ones that Bolton and Gillett invoke, we can cite the enthusiasm for operationalism in midtwentieth century philosophy of science that, some believe, entered the psychiatric discourse by way of a talk to the American Psychopathological Association by the logical empiricist Carl Hempel in 1959 (Hempel 1994). This is a case, though, that shows the complexity of establishing philosophical influence; the idea that Hempel caused the APA to immediately pivot to a new approach for the DSM-III has been debunked (Fulford and Sartorius 2009; Schaffner and Tabb 2014; Aragona 2015). Taking this episode as a cautionary tale, Blashfield and Cooper (2018) have argued that philosophers can be lulled into creating origin myths about their own field - philosophy of psychiatry - which in fact exaggerate the influence of philosophy on psychiatry, for the obvious reason that it is validating. At the same time, it is clear that the language of operationalism was taken to be germane both by philosophers and by psychiatrists themselves, such that it was useful as a means of characterizing shifts that were already underway (Tekin 2019). My sense is that something similar has happened with Cartesian dualism, on a grander scale.

In any event, I believe the most significant conceptual vectors of contemporary psychiatry's development to be more recent and more mundane. I will discuss two in this section: intradisciplinary specialization, and market pressures favoring translational research (that is, research that applies basic science findings to medical therapeutics) over clinical 
research (that is, original research on human subjects). Each of these vectors has contributed to the contemporary moment, in which the unity of psychiatry's different constituencies-clinicians, researchers, and patients - is at a nadir. The dramatic rift between the National Institute of Mental Health (NIMH) and the American Psychiatric Association (APA) in the early 2000s, brought on by the NIMH's introduction of an alternative to the DSM for researchers, brought these tensions into explicit view. This alternative, the Research Domain Criteria matrix (RDoC), did not aim to replace the DSM in clinical contexts - if it did, it would have been a centripetal force, not a centrifugal one. Rather, the NIMH sought to break what Steven Hyman has called the "epistemic bottleneck" that the clinical conceptual framework imposes on the research setting. Hyman lamented that research questions were neglected when they crosscut the DSM's diagnostic categories, because of the challenge of finding causal mechanisms in heterogenous research samples (Hyman 2010; for discussion see Tabb 2015). When he took over the NIMH's directorship from Hyman in 2002, Thomas Insel (2014) zealously ushered in not only $\mathrm{RDoC}$ but also a new vision of psychiatry as "clinical neuroscience".

The introduction of $\mathrm{RDoC}$ was significant because it aimed to sever one of the main centripetal forces acting on psychiatry: the hold the DSM had over both clinicians and researchers. The fractious relationship of those working in and around psychiatry to the DSM was already well established. Theorists have noted that clinicians themselves have for decades used the manual less as a scientific guide for understanding psychopathology than as a codebook for managing insurance reimbursements (First and Westen 2007; Whooley 2010). And indeed many clinicians do not need a scientific guide; their work is about setting clients up with social services and managing care, including medications which are prescribed on the basis of inductive expertise at best and trial and error at worst. Although it has gotten less attention, it is notable that during the same years RDoC was developed, psychoanalytically-oriented clinicians went so far as to adopt their own manual, the Psychodynamic Diagnostic Manual, out of frustration with the DSM. More recently a large coordinated effort to offer a new psychologically-grounded alternative to both the DSM and RDoC has taken off, called the Hierarchical Taxonomy of Psychopathology Consortium (HiTOP) (Kotov et al. 2017).

While the NIMH's introduction of RDoC has been taken as a declaration of war against the DSM, this broader context suggests it may go the other way: specialization within the field has made it harder for the DSM, regularly referred to as psychiatry's "bible," to work for everyone amidst the mounting schisms (Lilienfeld 2014). As the complexity of mental illness has emerged with advances not only in the basic sciences but also 
in fields like epidemiology, sociology, and human rights, the need for care teams that bring together experts with very little overlap-such as social workers and geneticists - comes ever more into view. The strain put on the DSM to be of use to all these constituencies has been enormous, unparalleled by most other diagnostic instruments (Kutschenko 2011a). Nonetheless, it is hard to imagine something replacing the DSM's crucial role as what Lara Kutschenko Keuck has called an "epistemic hub", facilitating "large-scale interactions without necessarily providing a complete infrastructure". According to Keuck, broadly applied classification systems like the DSM "can be regarded as important nodal points for various actors in biomedical and epidemiological research, clinical practice, and public health" (Kutschenko 2011b, 594). When the hub cracks, the spokes fly loose, and the wheels begin coming off the wagon.

Given all this, the fact that the NIMH decided it advisable, even possible, to do psychiatric research without appeal to the constructs clinicians use to diagnose and treat patients shows how far specialization has come within psychiatry. About the growing gulf between the different constituencies working in and around psychiatry there is much to say, and happily we have historians to say it (see, for example, Halliwell 2013; Menninger and Nemiah 2000; Shorter 1997). From the swelling ranks of case workers to the dwindling ranks of psychoanalysts, the evidence points to these changes being explicable mainly in terms of twentieth-century developments in economics, in labor, and in social policy, rather than as a result of a resurgence of dualist or physicalist commitments. The swing of the pendulum over the course of the twentieth century between the psychoanalytic era's emphasis on early childhood experience, memory, and psychodynamics to the biomedical emphasis on functions, dysfunctions, and physiology does not correspond to any contemporaneous movement in philosophy, whose own "mechanistic revolution" came centuries earlier. Within psychiatry, reductionism - that is, the favoring of explanations that focus on causal relationships between wholes and their constituent parts - was on the rise in psychiatry in the 20th century, but whether it precipitated or resulted from specialization is not obvious. What is clear is that the increasing silos of biomedical research, clinical research, and clinical practice, and the increasing breakdown in interaction between the specialists working in each, has been accompanied by a growing prioritization of basic science and translational research within the field. Biomedicalism is winning.

Members of the American Psychological Association recently sounded the alarm about the NIMH's shift towards "clinical neuroscience" in an open letter to the DSM-5 task force, writing "In light of the growing empirical 
evidence that neurobiology does not fully account for the emergence of mental distress, as well as new longitudinal studies revealing long-term hazards of standard neurobiological (psychotropic) treatment, we believe these changes [in favor of biological description] pose substantial risks to patients/clients, practitioners, and the mental health professions in general" (Kamens et al. 2017). The sense among psychologists, social workers, epidemiologists, and other researchers that the NIMH was deprioritizing their research in favor of basic science and translational research has been recently verified empirically (Teachman et al. 2018). These repercussions are rippling far beyond the NIMH itself and other government agencies; for example, Schwartz et al. (2016) note that psychology departments are increasingly changing their names to sound more biological, often by adding the word "neuroscience". Karina Stone and colleagues have demonstrated, using a literature review of articles published in 2008, that about half of all articles in the two major psychiatric journals-American Journal of Psychiatry and The Archives of General Psychiatry-in that year treat biological themes, as opposed to epidemiological, clinical or review treatment studies (Stone 2012). Strikingly, this percentage was far higher than in leading internal medicine journals, where the number of biologically-oriented papers was only $22 \%$. Psychiatry has become a less hospitable field for those doing clinical, as opposed to biomedical, research.

Bolton and Gillett themselves take an optimistic view of the NIMH's new orientation, suggesting that $\mathrm{RDoC}$ could act as a centripetal force insofar as "it could be elaborated in various ways to have broader scope appropriate for the biopsychosocial model" $(2019,128)$. This sort of elaboration is where their model really shines. By defining the sphere of psychiatry as an entangled systems of regulatory control mechanisms that span a broad scale, from the molecular architecture of organic matter to the individual making choices in response to their environment, Bolton and Gillett show how the limitation of psychiatric inquiry to certain levels of analysis will impoverish the field. Their ambitions for RDoC include the integration of health conditions pertinent to mental functioning, as well as attention to the stages of disease progression and maintenance, and the inclusion of population as opposed to just individual-level information. Their discussion shows how their framework has the potential to guide the expansion of the RDoC matrix beyond its current constructs and domains, which are drawn quite narrowly from cognitive neuroscience. It could give principled grounds for expanding the NIMH's vision of psychiatric research to address the concerns of those researching causal pathways that, while nonbiological, are no less legitimate scientific targets. 
Despite the power of Bolton and Gillett's model and the ease with which it could be applied to expand the matrix for future iterations of RDoC, I find it unlikely that the NIMH will be tempted. This is because the NIMH's commitment to reductive explanations does not come from an underexposure to metaphysics, but rather from market pressures that favor certain levels of medical explanation over others. While Bolton and Gillett present $\mathrm{RDoC}$ as open to a biopsychosocial approach because of its range of levels of analysis $(2019,126)$, the highest level of the current matrix is patient self-report - there is no place for social or environmental factors. This is because RDoC was envisioned quite explicitly as psychiatry's debut within the new "precision medicine" paradigm, a hugely influential global push by governments and private research and development institutes to reorient biomedical research towards viable pharmaceutical targets (consider, for example, the title of Insel's 2014 paper in the American Journal of Psychiatry, "The NIMH Research Domain Criteria Project: Precision Medicine for Psychiatry"). In line with these broader precision aims, RDoC's architects have stated explicitly, through a series of "postulates," that the matrix is intended to prioritize neurobiological explanations over other levels of analysis:

First, mental illnesses are presumed to be disorders of brain circuits. Secondly, it is assumed that the tools of clinical neuroscience, including functional neuroimaging, electrophysiology, and new methods for measuring neural connections can be used to identify dysfunction in neural circuits. Third, the RDoC approach presumes that data from genetics research and clinical neuroscience will yield biosignatures that will augment clinical signs and symptoms for the purposes of clinical intervention and management. (Morris and Cuthbert 2012, 33)

Rather than the specter of post-Cartesian thought, I believe that the NIMH's shift towards neuroscience is motivated by the same factors as the shift towards genetic research in precision medicine writ large. The development of psychopharmacology has stalled horribly, and as a result the drug industry has lost interest in researching new treatments for the DSM's diagnoses - they don't pay. The dramatic success of precision medicine drugs in other fields (for example Herceptin, an effective treatment for cancers that are HER2 receptor positive) has revived hope among biomedical researchers that a turn away from signs and symptoms and towards molecular biomarkers will be transformative. About this, too, I am skeptical (see Lemoine and Tabb, forthcoming), but it seems undeniable that the NIMH's attempt to pry biomedical psychiatry free from the conceptual strictures of the clinic follows along from the economic 
realities facing its researchers. It seems doubtful a philosophical intervention alone could counter the centrifugal forces of the market, which are pushing clinical research that is deemed profitless to the periphery.

\section{The Centripetal Power of Ethical Principles}

Building on the previous section, I argue here that if my analysis of psychiatry's current centrifugal pressures is correct, it follows that the best way to address them is not merely through the introduction of a new ontology, but through also making a normative case for the value of such an ontology. I have suggested that the competition for limited resources has driven the split between biomedical psychiatry and clinical psychiatry - the two have been pulled apart not, I have argued, because of entrenched dualism, but because of market forces. There has long been confusion about psychiatry's self-image, with some of its practitioners seeing it as applied neuroscience, some as applied psychology, some as a social welfare project, some as a humanistic quest, etc. But a shortage of resources means that a thousand flowers cannot bloom. While a more inclusive ontology such as that proposed by Bolton and Gillett would refocus psychiatry's scattered attention through its top-down emphasis on the person, its adoption would need to be justified for researchers whose careers have been shaped by centrifugal pressures towards specialization. For many psychiatrists, the disaggregation of biomedical research from clinical practice makes their work possible.

Importantly, such disaggregation is also compatible with a commitment to a fundamentally unified biopsychosocial ontology. Given psychiatry's division of labor, a researcher can recognize the reality of the psychological and social aspects of mental illness but ignore them during a day's work in the lab. In other words, while the biopsychosocial framework seeks to remind biomedicine of its need for psychological and social components on the grounds of ontological entanglement, given the successes of neurobiology in explaining cognition from within a reductionist frame, and the current trends in federal and private funding, this is a hard case to make. Furthermore, while the adoption of a new biopsychosocial ontology would give a rationale for a more evenhanded approach to psychiatric research at the structural level-encouraging funding of both biomedical and psychosocial investigationshandwringing about the exclusion of the psychosocial has not, so far, been effective at countering the powerful centrifugal motion stirred up by increasing investment in the lucrative promise of precision psychiatry. 
Using Bolton and Gillett's $(2019,121)$ language of "modifiable causes", that is, promising targets for intervention, we can say that apologists for the NIMH's neurocentrism are favoring causes operating at the neuroscientific level because they seem the most rewardingly modifiable. Here are Cuthbert and Kozak, for example:

[I]t is clear that a diagnostic system based upon empirical data from genetics, neurobiology, and behavioral science is desirable to move toward an era of precision medicine where patients are diagnosed and treated according to accurate and appropriately fine-tuned assessments. (Cuthbert and Kozak 2013, 929)

Their emphasis on the applied sciences is pragmatic, not philosophical. It seems the NIMH could very well acknowledge the rich ontology of psychiatry's objects and still insist that some are more worth investigating; the point of RDoC is precisely that biomedical psychiatry does not need clinical psychiatry to point out the appropriate targets for scientific investigation. While Bolton and Gillett are surely right that "it is of fundamental importance in healthcare [that w]e attend to the person, not the body part-and not to psychological signs and symptoms in isolation either" $(2019,116)$, the fundamental importance of the person to the biomedical researcher is less obvious, given psychiatry's extensive specialization.

As resources shift towards the most powerful interest groups in psychiatry - those with the capital to invest in innovation - and away from those at the less glamorous front lines of mental healthcare (such as social workers, therapists, and general practitioners) there are not only philosophical but practical repercussions. Ethical arguments attending to these repercussions have the potential to bring critical attention from a large range of stakeholders. On ethical grounds one can question whether people's basic rights to healthcare are best served by a psychiatry reconceived as clinical neuroscience (Kirmayer and Crafa, 2014); whether medicine driven by powerful economic interests will align with best bioethical practices (Jeungst et al. 2016); or whether discoveries in neuroscience or genetics, funded by tax-payer dollars, are liable to translate into transformative medical treatments any time soon (Tabb 2020). These questions cannot be brushed aside on the grounds that psychiatric biomedicine is doing just fine without the psychosocial, because they question what "just fine" really amounts to. Questions like these implicate not just to those trying to do good science or provide effective care, but also those who use the mental healthcare system, or even just pay taxes. 
Their answers require top-down thinking, not with respect to levels of ontological complexity, but with respect to our higher-order ethical commitments from which decisions about care are deduced. Joseph Margolis has argued that

medicine is ideology restricted by our sense of the minimal requirements of the functional integrity of the body and mind (health) enabling (prudentially) the characteristic activities and interests of the race to be pursued. (Margolis 1976, 253)

These prudential interests should not, Margolis emphasizes, be confused with the natural functions of the human organism, nor even with the generic values of rational agents. We must attend to the "ulterior goals of given societies" that "reflect the state of the technology, the social expectations, the division of labor, and the environmental condition of those populations" (Margolis 1976, 252). Elsewhere I have argued that while our moral reasoning about such questions relies on empirical facts, it cannot be reduced to them (Tabb 2020). The empirical facts-facts like how transformative funds spent on basic research will be to future healthcare advances, or when these payoffs will come-rely on our understanding of causes, mechanisms, and systems. But only a broader ethical lens can bring into focus what we should do in response to these facts.

I am not the first to worry that without a unified ethical framework, an expansion of medicine's explanatory projects may only contribute to its dissolution. Moving beyond the case study of psychiatry, in the fractious scholarly debates over the value of precision medicine, critics from a variety of disciplines have expressed worry that the race to disrupt the medical industry with new discoveries can cause resultant healthcare inequities to be obscured. As Ron Bayer and Sandro Galea have written,

Research undertaken in the name of precision medicine may well open new vistas (...). But the challenge we face to improve population health does not involve the frontiers of science and molecular biology. It entails development of the vision and willingness to address certain persistent social realities, and it requires an unstinting focus on the factors that matter most to the production of population health. (Bayer and Galea 2016)

The payoff for the grinding work of addressing longstanding healthcare inequities and failures in the mental healthcare system is far from 
immediate, and therefore the research that would support it is disincentivized within a free market.

Roberto Lewis-Fernández and his coauthors have made similar observations in the context of mental health research, arguing that the shift towards basic and translational research in psychiatry risks neglecting

thorny details, such as what proportion of the budget should be allocated to what research areas; the near-term public health consequences of particular priorities; and how to leverage inter-agency collaborations to attain a robust and sustainable public health impact. (Lewis-Fernández et al. 2016, 509)

Given that the NIMH is the most significant source of public funds for psychiatric research in the United States, in the American context funding is something of a zero-sum game. In the decade surrounding RDoC's introduction, funding for clinical trials was cut by about a third; the Division of Services and Intervention Research and the Office of Research on Disparities and Global Mental Health was cut by almost 17\%; and spending on basic neuroscience went up by $28 \%$ (Insel 2015).

What would foundational principles be that could help us navigate these bioethical challenges? They might draw on common understandings of medicine's ultimate aims to give grounds for championing some sorts of medical endeavors over others. A reason to advocate against the centrifugalism of precision medicine, for example, could be that one believes medicine to be more beholden to patients than scientific projects of discovery. Margolis believes medicine to be "primarily an art, and, dependently, a science: it is primarily an institutionalized service concerned with the care and cure of the ill and the control of disease" (1976, 242), for which biological understanding is useful but not essential. Under such a view, funding bodies would have an obligation to make sure that any basic science research they fund has clear clinical application. Now of course immediately, longstanding ethical challenges jostle for attention - is it better to deliver imperfect care to patients in need now than to focus on transforming care options for future generations? Does society have an obligation toward the "worried well"- - that is, to manage the daily stress of life? Insofar as it can be argued that poverty is a leading cause of mental illness, should the purview of mental health policy extend to questions of social welfare distribution? Etc. Developing worthwhile ethical principles to populate an ethical biopsychosocial model would take the same keen attention to our best bioethics, public policy, and political theory that Bolton and Gillett have paid to our best contemporary theories of causation and ontology. 
A more generous metaphysics that includes factors like personal agency is certainly friendlier towards this kind of ethical project than one which dismisses agency as epiphenomenal. But as a unifying framework, the biopsychosocial model has traditionally lacked the specificity to structure these medical-ethical debates. In other words, it has failed to provide an account of Margolis' prudential functions, those capacities that we prioritize not because they are natural to us but because they allow us to live in the ways we deem right. Whether to prioritize resolving LewisFernandez et al.'s "thorny details" or instead to attend to the fascinating puzzles of basic neuroscience or behavioral genetics cannot be answered on the basis of a pluralist ontology alone. Insofar as the whole personfrom genes to environmental interactions-is implicated in these questions, the biopsychosocial model offers no grounds for resolution. However, Bolton and Gillett argue explicitly that their model also holds a place for ethics within its ontology, in so far as it follows from agency being "thoroughly biological" (138) that it "becomes involved with morality," due to the entanglement of the biological, psychological, and social (88). Before closing I want to consider whether the theoretical ethical principles I am looking for "fall out" of their model in some way that would render the addendum I am proposing unnecessary.

\section{The Normativity of the Biopsychosocial Model}

Seeing RDoC as a wedge to move the basic and translational sciences towards the core of the discipline can explain why its advocates have ignored another repercussion of their attempted coup against the DSM: the loss of a bellwether for distinguishing the normal from the pathological. The architects of RDoC have shown little interest in taking up the mantel, emphasizing that they are merely interested in the elucidation of mechanisms, not in the demarcation of disease categories. But which mechanisms count as psychiatric? This is not just about semantics; the NIMH's mission is to fund research into mental health, not physiology, and $\mathrm{RDoC}$ is to a large degree about shaping what research counts as what (Tabb 2020). Without some grounds for ruling on what counts as psychiatric and what doesn't, the NIMH can increasingly fund basic research in, e.g., neuroscience or genetics, moving the institute ever further away from its traditional focus on mental illness as a societal problem (Bloom 2002, 165).

Insel, writing with Bruce Cuthbert, has suggested that maybe mental disorders can be defined as extremes of functional variation, writing, 
The idea [of RDoC] is to start by specifying basic dimensions of functioning, and their implementing brain circuits, that have been identified by the last several decades of research in brain and behavior. Then, in this light, mental disorders are considered as extremes at one or both tails of those normal distributions. (Cuthbert and Insel, 2010, 312)

This approach to delineating diseases - as tails on a normal distributionis profoundly unsatisfactory, as philosophers of medicine have long pointed out (Boorse 2011, 21). Which tail (one or both)? Where is the cutoff (and who decides)? Jerome Wakefield has described RDoC's naïve approach to the demarcation problem as a failure of conceptual validity. "Whatever its errors," Wakefield writes, the DSM

remains an attempt to delineate the domain of psychological conditions that fall under the concept of disorder. RDoC offers nothing to replace the [DSM's] efforts to delineate the domain of disorders and provide a target at which construct validation can aim. (Wakefield 2014, 38)

The results are "so weak that it is difficult to envision success" (ibid.).

Broadly speaking, attempts by philosophers and psychiatrists to provide an analysis of mental disorder that could help demarcate psychiatry's objects have been copious, heated, and ultimately inconclusive (for recent moves in this debate see Faucher and Forest 2021; for a critical analysis of it see Lemoine 2013). Bolton and Gillett themselves offer a hybrid view, combining naturalist and normativist elements, in which they argue that normativity "is fundamental to biological regulatory control mechanisms" $(2019,68)$ and that therefore disease can be understood, generally, in terms of failures of function produced by these feedback mechanisms. They suggest that the levels of dysfunction where mental pathology manifests in practice - the psychological and the social-are emergent manifestations of these biological dysfunctions $(2019,72)$. However, on the grounds of their comfort with top-down causation, they also suggest that dysfunction can be located in any part of a system that is both modifiable and the cause of error: "From this point of view, dysfunction attribution is in part-and somewhat paradoxically_shorthand for belief about promising possibilities for change" $(2019,121)$. The need to change, they suggest elsewhere, comes with patients' self-report of "distress: with worry and fear about their safety and their future and their dependents" (135).

Demarcations between the normal and the pathological that rely even in part on naturalist theories of dysfunction have, to my mind, been 
convincingly problematized by philosophers like Ron Amundson, who have argued that the ontological makeup of the individual organism can shed light on mode of function, but not establish level of function. Writing in the context of disability, Amundson argues that what matters for defining disability is an individual's capacities within a given environment; their functional makeup is irrelevant to determinations of health. "If we thought merely about level of functional performance, rather than mode, fashion, or style of function," Amundson writes, "the disadvantages of disability would not seem so natural and inevitable" $(2000,48)$. Amundson's case for rejecting biological theories of dysfunction is also an ethical one-to focus on mode is to facilitate the continuation of historic abuses against those who function differently.

Bolton and Gillett recognize disabilities as "a special case" due to the lack of modifiable causes within organism's system, allowing that here errors "can be legitimately attributed to (...) external factors" (114). While they insist that "disability related concepts and practices involve a complex range of and interaction between biological, psychological, social, moral and policy factors" and therefore "cannot be so much as articulated without a full biopsychosocial framework," it is unclear on what grounds their new ontology - reliant as it is on locating dysfunction within the system-could offer robust support to a social model of disability like Amundson's, which takes the black-boxing of function, and a turn to the disabling features of the environment, to be an ethical imperative. At one point in their book, Bolton and Gillett seem to accept that while generally they are committed to locating "the problem - the dysfunction-in the person", they must make an exception for conditions that are lifelong and/or not amenable to change $(2019,120)$.

The fact that the new biopsychosocial framework has little to offer on these conditions should give us pause, given the percentage of mental disorders that display them. Furthermore, those diagnosed with psychiatric disorders are increasingly conceptualizing their conditions in terms of difference rather than dysfunction, in alignment with the social model of disability. While there has always been robust activism in response to the perceived overreach of biomedical psychiatry, contemporary activists have introduced a new conceptual framework for thinking about this resistance. Instead of denying that purported mental illnesses have any clinical relevance, like the radical antipsychiatrists of 1960s and 70s, some contemporary critics argue for destigmatization alongside new demands for healthcare justice. To advocate for neurodiversity is to believe that healthcare, social services, and culture broadly construed must change to offer a broader range of supports, allowing not only the neurotypical but the neurodiverse to flourish. To be neurotypical, in other words, is just to 
have the sort of psychological profile that is already served (more or less) well by one's environment, and there is no reason to see such a profile as innately healthier, rather than just more convenient under the current circumstances. Given the neurodiversity movement's suspicion of essentializing ontologies, its reliance on social constructionist narratives of illness, and its impatience with biomedical levels of description, its best ontological allies may be quietist, not pluralist. What would really help is a psychiatric ethics capable of justifying their claim to healthcare as a human right, even in the absence of dysfunction.

\section{Final thoughts}

I have argued that the centrifugal forces causing rends in psychiatry's conceptual fabric are due to a confluence of political, economic, and cultural factors. The displacement of the DSM as the field's arbiter of the normal and the pathological was both a result and a driver of increased specialization within the field, which led to new antagonisms and struggles. The economic promise of the precision medicine model, which matches patients with novel therapies on the basis of biomarker testing, has caused an influx of financial support for biomedical approaches to psychopathology. Advocates of precision psychiatry need not deny that there are other levels on which psychopathological phenomena can be found, and intervened upon-such as the psychological or the social. But they may doubt that there are modifiable causes to be found at these levels, or that these causes are as rewardingly modifiable as those found at the level of the neural circuit. While Engel wished psychiatrists to be "concerned primarily with the study of man and the human condition" (1992, 327), this hardly seems realistic for the twenty-first century biomedical researcher, whose lab work in psychiatric genetics or in neuroscience may never require meeting a patient.

The result of this recent enthusiasm for precision psychiatry is that the field is increasingly pulled in different directions. Its practitioners rely on traditional disease categories as well as their own expert knowledge of psychopathology to do their work, while its researchers borrow the concepts and methods of the basic sciences for theirs. Similar changes are underway in other fields where the precision paradigm has taken hold. To counter this centrifugal motion, I have suggested, a new ontology is not enough, because the motivations for the split do not result from monist or reductionistic ontological commitments as much as they do from economic and political factors. These systemic pressures on the profession force different sorts of practitioners farther apart, and reward psychiatric research that diffuses its center of gravity away from immediate mental 
health crises. Accordingly, to convince the diverse stakeholders in psychiatry that it is important to all work toward the same thing, ethical arguments hold greater promise. They can exert pressure on the powers making decisions about what kind of psychiatric research is worth funding, and what kind of mental healthcare is worth expanding. A new ontology that takes seriously the complex feedback loops between the biological, the psychological, and the social has the potential to encourage a revaluing of neglected populations. But the need to adopt such an ontology may only become clear when it is shown how the exclusion of psychosocial dimensions causes us to fail in our ethical obligations.

It is worth noting that the biopsychosocial model itself might be conceived of in purely prudential terms, instead of in metaphysical terms. Such a theory would offer a model of psychiatry as unified by the biological, psychological, and social aspects of people's mental health, not because these are aspects of a unified ontology, but because they form a unified set of obligations. In her "Neurodiversity at Work: A Biopsychosocial Model and the Impact on Working Adults," Nancy Doyle notes that the biopsychosocial model can be maintained even amidst "ontological controversy" over the nature of mental illnesses like autism. She glosses its biological component as "therapeutic intervention" rather than as referring to any (dys)function within the individual, and the model as a whole is taken as a pragmatic one, with the explicit aim of realizing the best outcomes for neurodiverse people in the workplace (119). By dismissing concerns about the place of the pathological, however, this account is ultimately centrifugal, disaggregating the question of how neurodiverse people should be treated in the workplace from larger ones concerning psychiatry's biomedical projects.

In contrast, Bolton and Gillett's new biopsychosocial model is exciting for its stout centripedalism, which could ground an ethical framework for all of medicine. Yet as it currently stands, the model does not contain foundational principles capable of negotiating, on ethical grounds, between those advocating for biological, psychological, or social approaches to disease. It is this nonpartisan tendency of the biopsychosocial model that has, I think, frustrated critics. This reflects a broader suspicion about pluralism: that one can end up with a conglomerate of models that, taken together, are like the map in Borges' story "On Rigor in Science". Cartographers render this map so exact that it papers over the whole land, rendering itself useless. One feels for Bolton and Gillett when their amendments of the $\mathrm{RDoC}$ matrix cause it to grow rather threateningly, in their words, into "a multidimensional monster grid" (130). The authors encourage us to see this complexity and uncertainty as a result of the science itself, rather than the model - "no point in blaming the messenger" 
(132). But the moral of Borges's story is that the main responsibility of the modeler lies precisely in picking the right scale for the job. A scientific theory is, in this analogy, not the messenger but the message itself, which aims to render legible the complexity of the modeled system. Which "aetiology of small effect" (132) we take as definitional of health conditions must be made not only by "doing science" but also by making choices between modifiable causes. As Bolton and Gillett note, medicine is an "applied science, seeking to change things, for the better" $(2019,121)$. If so, the explanatory choices that result from a model should be normative. Determining what differences in function are appropriate targets for medical intervention and which are better left for scientific or societal interventions cannot be read off the individual's own state of functioning or agential status. It relies on broader societal norms concerning wellbeing, and the ethical commitments of medicine itself.

I believe that general ethical principles could be addended to the new biopsychosocial model without requiring it to give up its neutrality with respect to the relative value of the biological, the psychological, and the social. Instead, the framework could host a normative pluralism analogous to the ontological pluralism undergirding the "multiple specific biopsychosocial models" that Bolton and Gillett allow for, in which the relevance of each aspect will change depending on prudential functions relevant to the case at hand. At the same time, the model could seek to supply the abstract theoretical constructs necessary for a powerful new medical ethics. Being integrated into the new biopsychosocial framework would assure that these theoretical constructs would guide all research and practice falling under the broad reach of the model.

\section{REFERENCES}

Aragona, Massimiliano. 2013. 'Neopositivism and the DSM Psychiatric Classification: An Epistemological History'. Part 1: Theoretical Comparison'. History of Psychiatry, 24: 166-179.

Bloom, Samuel William. 2002. Word as Scalpel. Oxford: Oxford University Press.

Bolton, Derek, and Grant Gillett. 2020. The Biopsychosocial Model of Health and Disease: New Philosophical and Scientific Developments. Cham, Switzerland: Palgrave Pivot.

Cooper, Rachel, and Roger Blashfield. 2018. 'The Myth of Hempel and the DSM-III'. Studies in the History and Philosophy of Biology: Biomedical Sciences. 70: 10-19. 
Cuthbert, Bruce, and Thomas Insel. 2010. 'The Data of Diagnosis: New Approaches To Psychiatric Classification.' Psychiatry, 73 (4): 311-314.

Cuthbert, Bruce, and Michael J. Kozak. 2013. 'Constructing Constructs for Psychopathology: The NIMH Research Domain Criteria'. Journal of Abnormal Psychology 122 (3): 928-937.

Engel, George. 1992 [1977]. 'The Need for a New Medical Model: A Challenge for Biomedicine'. Reprinted in Family Systems Medicine, 10 (3): 317-331.

First, Michael B, and Drew I Westen. 2007. 'Classification for Clinical Practice: How To Make ICD And DSM Better Able To Serve Clinicians'. International Review of Psychiatry 19: 473-481.

Fulford, K. W. M., and Norman Sartorius. 2009. 'The Secret History of ICD and the Hidden Future Of DSM'. In Psychiatry as Cognitive Neuroscience: Philosophical Perspectives, edited by Matthew Broome and Lisa Bortolotti, 151-158. Oxford: Oxford University Press.

Galea, Sandro and Ronald Bayer. 2016. 'The Precision Medicine Chimera'. Project Syndicate. January 14.

Ghaemi, S. Nassir. 2010. The Rise and Fall of the Biopsychosocial Model: Reconciling Art \& Science in Psychiatry. Baltimore: Johns Hopkins University Press.

Halliwell, Martin. 2013. Therapeutic Revolutions: Medicine, Psychiatry, and American Culture, 1945-1970. Rutgers University Press.

Hempel, Carl. 1994. Fundamentals of Taxonomy. In Philosophical Perspectives on Psychiatric Diagnosis Classification, edited by John Z. Sadler, Osborne P. Wiggins, \& Michael A. Schwartz, 315-332. Baltimore: Johns Hopkins University Press.

Hyman, Steven E. 2010. 'The Diagnosis of Mental Disorders: The Problem of Reification'. Annual Review of Clinical Psychology 6: 155179.

Insel, Thomas R. 2015. "Anatomy of NIMH Funding," available at http://www.nimh.nih.gov/funding/fundingstrategy-for-researchgrants/the-anatomy-of-nimh-funding.shtml.

Insel, Thomas R. 2014. 'The NIMH Research Domain Criteria (RDoC) Project: Precision Medicine for Psychiatry'. American Journal of Psychiatry 171 (4): 395-397.

Insel, Thomas R., and Remi Quirion. 2005. 'Psychiatry as a Clinical Neuroscience Discipline'. Journal of the American Medical Association 294 (17): 2221-24.

Juengst, Eric, Michelle L McGowan, Jennifer R. Fishman, and Richard A. Settersten Jr. 2016. From "Personalized" to "Precision" Medicine: The Ethical and Social Implications of Rhetorical 
Reform in Genomic Medicine. Hastings Center Report 46: 2133.

Kamens, Sarah R., David N. Elkins, and Brent Dean Robbins. 2017. 'Open Letter to the DSM-5', available online at:

https://www.ipetitions.com/petition/dsm5/.

Kirmayer, Laurence J., and Daina Crafa. 2014. 'What Kind of Science for Psychiatry?'. Frontiers in Human Neuroscience 12: 1-12.

Kotov, Roman, Robert F. Krueger, David Watson, et al. 2017. The Hierarchical Taxonomy of Psychopathology (Hitop): A Dimensional Alternative to Traditional Nosologies. Journal of Abnormal Psychology, 126 (4): 454-477.

Kutschenko, Lara K. 2011a. 'In Quest of 'Good' Medical Classification Systems'. Medicine Studies 3: 53-70.

Kutschenko, Lara K. 2011b. 'How to Make Sense of Broadly Applied Medical Classification Systems: Introducing Epistemic Hubs'. History and Philosophy of the Life Sciences Part C, 33: 583-602. Lilienfeld, Scott. 2014 'DSM-5: Centripetal Scientific and Centrifugal Antiscientific Forces'. Clinical Psychology: Science and Practice 21 (3): 269-279.

Margolis, Joseph. 1976. 'The Concept of Disease'. Journal of Medicine and Philosophy 1 (3): 238-255.

Morris, Sarah E, and Bruce N. Cuthbert. 2012. 'Research Domain Criteria:

Cognitive Systems, Neural Circuits, and Dimensions of Behavior.' Dialogues in Clinical Neuroscience 14 (1).

Schaffner, Kenneth F. 1994. 'Psychiatry and Molecular Biology: Reductionistic Approaches to Schizophrenia'. In Philosophical Perspectives on Psychiatric Diagnostic Classification, edited by John Z. Sadler, Osborne P. Wiggins, \& Michael A. Schwartz. Baltimore: Johns Hopkins University Press. 279-294.

Schaffner Kenneth F., and Kathryn Tabb. 2014. 'Response to Josef Parnas'. In Perspectives in Philosophy and Psychiatry III: The Nature and Sources of Historical Change, edited by Kenneth S. Kendler and Josef Parnas. Oxford: Oxford University Press, 213220.

Schwartz, Seth J., Lilienfeld, Scott O., Meca, Alan, and Katheryn C. Sauvigné. 2016. 'The Role of Neuroscience Within Psychology: A Call for Inclusiveness Over Exclusiveness'. The American Psychologist 71, 52-70.

Shorter, Edward. 1997. A History of Psychiatry: From the Era of the Asylum to the Age of Prozac. John Wiley \& Sons.

Stone, Karina, Elizabeth A. Whitham, and S. Nassir Ghaemi. 2012. 'A Comparison of Psychiatry and Internal Medicine: A Bibliometric Study'. Academic Psychiatry 36 (2): 129-32. 
Tabb, Kathryn. 2015. 'Psychiatric Progress and the Assumption of Diagnostic Discrimination'. Philosophy of Science 82 (5): 10471058.

Tabb, Kathryn. 2020. 'Should Psychiatry Be Precise? Reduction, Big Data, and Nosological Revision in Mental Health Research'. In Levels of Analysis in Psychopathology edited by Kenneth S. Kendler, Josef Parnas, and Peter Zachar. Cambridge: Cambridge University Press, 308-334.

Teachman Bethany A., Dean McKay, Deanna M. Barch, Mitchell J. Prinstein, Steven D. Hollon, and Dianne L. Chambless. 2019. 'How Psychosocial Research Can Help the National Institute of Mental Health Achieve Its Grand Challenge to Reduce the Burden of Mental Illnesses and Psychological Disorders'. The American Psychologist 74 (4): 415-431.

Tekin, Şerife. 2019. 'The Missing Self in Scientific Psychiatry'. Synthese 196 (6): 2197-2215.

Whooley, Owen. 2010. 'Diagnostic Ambivalence: Psychiatric Workarounds and the Diagnostic and Statistical Manual of Mental Disorders'. Sociology of Health \& Illness 32 (3): 452-69. 